\title{
Research in Positive Psychology: a Systematic Literature Review
}

\author{
Caroline Tozzi Reppold - Universidade Federal de Ciências da Saúde de Porto Alegre, Porto Alegre, Brasil \\ Léia Gonçalves Gurgel - Universidade Federal de Ciências da Saúde de Porto Alegre, Porto Alegre, Brasil \\ Cecilia Cesa Schiavon - Universidade Federal de Ciências da Saúde de Porto Alegre, Porto Alegre, Brasil
}

\begin{abstract}
Resumo
A Psicologia Positiva aborda potencialidades e virtudes humanas, diferente da Psicologia tradicional que, até o fim dos anos 90 , considerava prioritariamente as questões de cura e reparo de danos. O objeto do presente estudo, por meio de uma revisão sistemática da literatura, foi apresentar o perfil dos estudos brasileiros em Psicologia Positiva. Percebe-se que a Psicologia Positiva no Brasil está em fase de expansão, mediante o aumento dos estudos publicados. Porém, apesar dos grandes avanços encontrados, a literatura nacional sobre o tema ainda apresenta lacunas importantes a serem sanadas, especialmente, em relação às intervenções voltadas para a Psicologia Positiva.
\end{abstract}

Palavras-chave: Psicologia Positiva, Brasil, bem-estar, esperança.

Pesquisas em Psicologia Positiva: uma Revisão Sistemática da Literatura

\begin{abstract}
Positive Psychology focuses on human virtues and strengths, in opposition to traditional Psychology, which, until the end of 90's, considered mainly issues regarding healing and damage repair. The aim of this study is to present the profile of Brazilian studies in Positive Psychology through a systematic literature review. It was found that studies in Positive Psychology in Brazil are still in their inception and gradually gaining recognition. However, despite a growing interest in this field of study, the Brazilian literature on this theme is still lacking in breadth and depth, especially regarding clinical interventions following the framework of Positive Psychology.

Keywords: Positive Psychology, Brazil, well-being, hope.
\end{abstract}

Investigaciones en Psicología Positiva: una Revisión Sistemática de la Literatura

\begin{abstract}
Resumen
La Psicología Positiva aborda la potencialidad y las virtudes humanas, a diferencia de la Psicología tradicional que hasta fines de los años 90, consideraba principalmente los temas de cura y reparación de daños. El objetivo de este estudio, a través de una revisión sistemática de la literatura, fue presentar el perfil de estudios brasileños en Psicología Positiva. Por medio del aumento de los estudios publicados se observa que la Psicología Positiva en Brasil está en fase de expansión. Sin embargo, a pesar de los grandes avances, la literatura nacional sobre el tema todavía presenta brechas importantes a ser consideradas, especialmente en relación a las intervenciones volcadas para la Psicología Positiva.
\end{abstract}

Palabras clave: Psicología Positiva, Brasil, bienestar, esperanza.

\section{Introdução}

A Psicologia Positiva, considerada como uma nova perspectiva no campo das ciências, tem sua origem controversa e parece estar relacionada com o início dos estudos sobre bem-estar subjetivo. Pereira (1997), em um panorama histórico-conceitual, aponta que essa abordagem teve início com os estudos de Terman, Jung e Watson, por volta da década de 1930. Nesse mesmo contexto, a Organização das Nações Unidas, por volta da década de 1950, passou a considerar, de maneira mais efetiva, outros níveis da vida das comunidades, como a qualidade de vida, a felicidade e o bem-estar (Pereira, 1997).

O termo "Psicologia Positiva" foi referido pela primeira vez por Maslow em 1954, em seus estudos sobre motivação e personalidade (Snyder \& Lopez, 2009). Porém, foi no final dos anos 1990 que Martin Seligman, nos Estados Unidos, legitima a utilização desse termo para denotar uma abordagem teórica proposta para a compreensão do ser humano, criando uma área específica de estudos junto à American Psychological Association (Seligman \& Csikszentmihalyi, 2000).

Seligman e Csikszentmihalyi (2000), em um artigo intitulado Positive Psychology: an introduction, sugerem que o campo da Psicologia, até o fim dos anos 90, considerava, prioritariamente, as questões de cura e reparo de danos, e apontam que atributos, como esperança, sabedoria, criatividade, coragem, espiritualidade, responsabilidade e perseverança estavam sendo ignorados pelos profissionais e pesquisadores da área. Citando essa lacuna no conhecimento, os mesmos autores 
sugeriram que, no século que se iniciava, os pesquisadores deveriam considerar, também, as forças e virtudes do indivíduo.

Assim, para Seligman e Csikszentmihalyi (2000), Psicologia Positiva é o estudo dos sentimentos, emoções, instituições e comportamentos positivos que têm a felicidade humana como seu objetivo final. Para Snyder e Lopez (2009, p. 17), Psicologia Positiva é a abordagem científica e aplicada para descobrir os pontos fortes das pessoas e promover o seu funcionamento positivo. Ainda, de acordo com esses autores, a ciência e a prática da Psicologia Positiva são direcionados para a identificação e compreensão das qualidades humanas e virtudes, bem como para promoção de condições para que as pessoas tenham uma vida mais feliz e mais produtiva. A partir dessa perspectiva, o significado da felicidade não é entendida como algo fugaz e flutuante, mas como um sentimento relativamente permanente experimentado ao longo do tempo (Scorsolini-Comin, Fontaine, Koller, \& Santos, 2013).

No ano de 2002, dando seguimento aos estudos voltados aos aspectos positivos dos indivíduos, Martin Selignam, em um documento intitulado Handbook of positive psychology (Seligman, 2002), refere que a Psicologia, portanto, deveria passar a ser vista, não apenas como o estudo da doença e dos danos, mas também de construtos, como a educação, o discernimento e o amor. O olhar, na Psicologia Positiva, deveria estar voltado, ainda, aos processos cognitivos e emocionais que facilitam mudanças comportamentais e o surgimento de estratégias de coping, além da promoção das potencialidades de cada sujeito (Barros, Martín, \& Pinto, 2010).

Nesse contexto, em seu primeiro livro sobre o tema, traduzido para o português com o título "Felicidade autêntica: usando a nova Psicologia Positiva para a realização permanente", Seligman (2004) salienta que a Psicologia Positiva se sustenta sobre três pilares principais, a saber: o estudo da emoção positiva, dos traços ou qualidades positivas e das instituições positivas (como a democracia, a família e a liberdade). No ano de 2011, um segundo livro foi publicado pelo mesmo autor, com o título "Florescer", do inglês Flourish. Nesse livro, considera-se que o tema principal da Psicologia Positiva é o bem-estar, e que o principal critério para mensurar esse construto é o "florescimento", sendo objetivo, das pesquisas e dos profissionais, aumentá-lo (Seligman, 2011).

Desde então, houve um aumento do interesse acadêmico e um emergente crescimento da Psicologia
Positiva. Hart e Sasso (2011), mapeando os contornos da Psicologia Positiva contemporânea, relatam um extraordinário crescimento na última década. Os autores, ainda, confirmam que os estudiosos nessa área têm dedicado a maior parte de sua atenção para dois dos três "pilares" da Psicologia Positiva, como proposto por Seligman e Csikszentmihalyi (2000): (1) o estudo da experiência subjetiva positiva e (2) as características pessoais positivas. Embora o interesse em instituições positivas ainda seja escasso, há a preocupação crescente com os temas da resiliência e da eudaimonia.

O Brasil, também, apresenta a Psicologia Positiva como um campo em expansão (Pureza, Kuhn, Castro, \& Lisboa, 2012). Em contexto nacional, o estudo da Psicologia Positiva teve seu início por meio de pesquisas sobre a resiliência e a vulnerabilidade dos sujeitos. O crescimento da área, também, foi marcado pela criação da Associação de Psicologia Positiva da América Latina (APPAL) em 2010 (Pureza et al., 2010) e da Associação Brasileira de Psicologia Positiva - ABP+, fundada em 2013.

Atualmente, pode-se afirmar que as pesquisas na área apresentam um crescente aumento, tanto em quantidade, quanto em qualidade (Araujo, 2013). O número de trabalhos, especialmente, aqueles com desenho metodológico teórico, como as revisões sistemáticas e integrativas, tem aumentado (Scorsolini-Comin \& Santos, 2010). Considerando as constatações mencionadas, é objeto do presente estudo, por meio de uma revisão sistemática da literatura, apresentar o perfil dos estudos nacionais, voltados aos temas relacionados à Psicologia Positiva, demonstrando a evolução dessa área do conhecimento e as lacunas nos estudos.

\section{Método}

\section{Estratégia de Pesquisa}

Foram pesquisadas as seguintes bases de dados eletrônicas, tendo como período janeiro de 1980 até outubro de 2014: MEDLINE (acessado pelo PubMed), LILACS, Psycinfo, SciELO, Scopus, Banco de Teses da Capes (Coordenação de Aperfeiçoamento de Pessoal de Nível Superior, fundação do Ministério da Educação do Brasil), além de referências adicionais que constavam na seção de referências bibliográficas dos estudos incluídos. Os termos de busca utilizados foram "Positive Psychology" e "Brazill", em português, inglês e espanhol e seus entre termos. 
Foram incluídos todos os estudos que tratavam sobre temas relacionados com construtos da Psicologia Positiva e que eram realizados no Brasil. Todos aqueles estudos que não apresentavam definição clara do conceito de Psicologia Positiva, que não definiam claramente o local de realização do estudo ou não contemplavam atributos dessa linha da Psicologia foram excluídos. Devido ao ineditismo e especificidade do evento, foram considerados também os anais do I Congresso Brasileiro de Psicologia Positiva, ocorrido em outubro de 2014, em Porto Alegre, RS.

\section{Análise dos Dados}

Os títulos e resumos de todos os artigos identificados pela estratégia de busca foram avaliados por dois pesquisadores independentes. Todos os resumos que não forneceram informações suficientes em relação aos critérios de inclusão e exclusão foram selecionados para avaliação do texto integral. No estágio do texto integral, dois revisores independentes avaliaram os artigos completos e realizaram suas seleções de acordo com os critérios de elegibilidade. Após, foi realizada a coleta de dados no que diz respeito às características metodológicas, intervenções e desfechos dos estudos utilizando formulários padronizados criados especificamente para o presente estudo.

As discordâncias, em todas as fases do processo de seleção, foram resolvidas por consenso entre os pesquisadores. Um terceiro revisor participou da etapa de decisão final sobre a elegibilidade de cada estudo. A análise dos estudos e dos resultados foi descritiva e as principais características dos estudos incluídos foram relatadas. Cabe ressaltar que, em relação aos anais do I Congresso Brasileiro de Psicologia Positiva, a análise dos dados se deu até a etapa de leitura dos resumos, já que não foram apresentados textos completos.

\section{Resultados}

Como resultado da busca inicial, foram identificados 42 estudos. Desses, 29 eram artigos oriundos das bases de dados pesquisadas, 12 eram dissertações de mestrado e o último era uma tese de doutorado. Ao final, foram incluídos 16 artigos das bases de dados, todas as dissertações e a tese (Figura 1). Os artigos excluídos tiveram como razão o fato de não apresentarem,

29 Citações potencialmente relevantes identificadas de todas as bases de dados, 12 dissertações e uma tese.

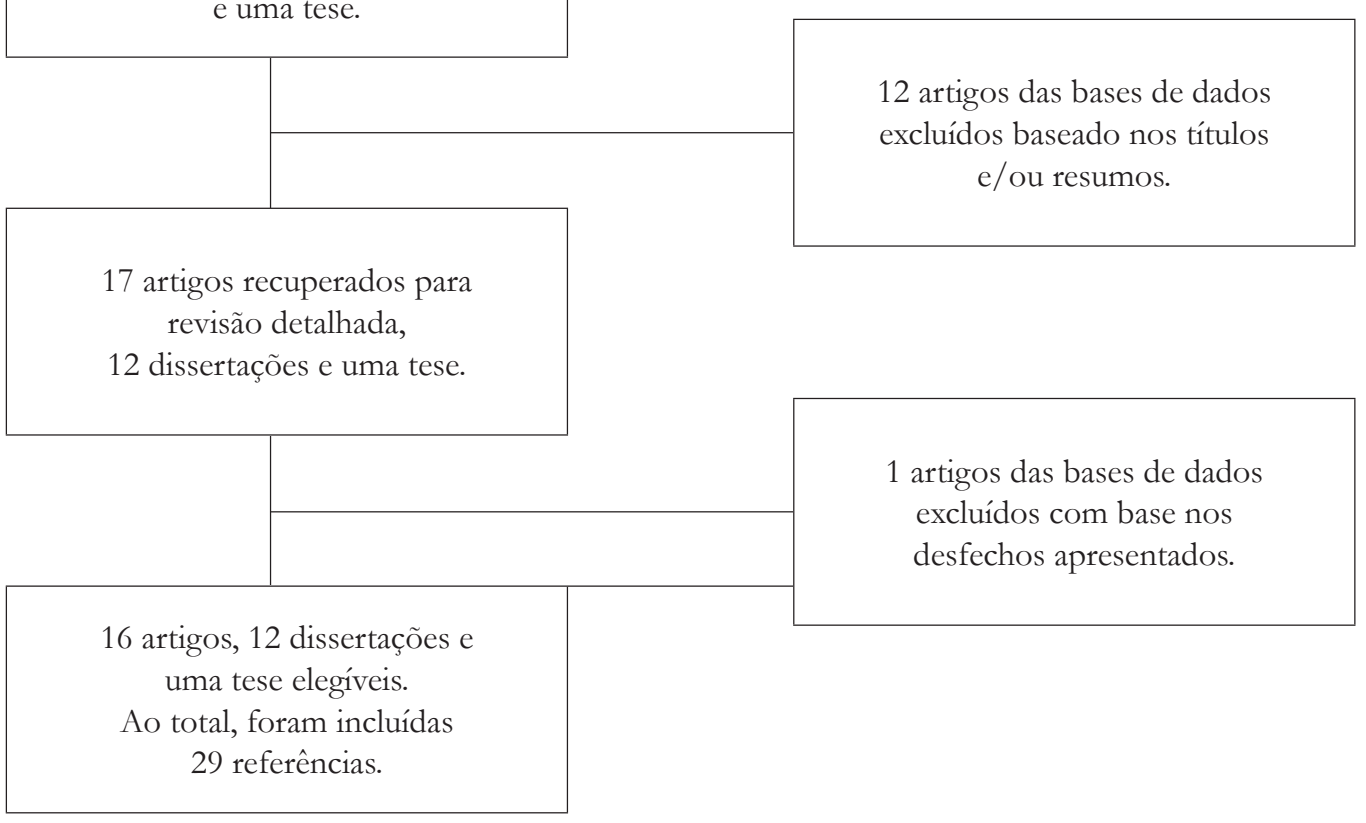

Figura 1. Fluxograma de seleção dos estudos completos incluídos. 
de forma clara e confiável, a metodologia ou o local de estudo, ou não contemplarem o tema proposto pela presente revisão. Na Figura 1, observa-se o diagrama de seleção dos estudos incluídos nesta revisão.

Todos os estudos incluídos foram publicados em português brasileiro, inglês ou espanhol, em periódicos nacionais e internacionais. Nenhuma das revistas apresentou fator de impacto. Em relação ao ano de publicação dos artigos encontrados nas bases de dados, observa-se que estiveram entre 2012 e 2013, com maior frequência de publicação no ano de $2013(n=4 ; 25 \%)$. As dissertações e tese, por sua vez, dataram apenas dos anos de 2011 e 2012.

Os estudos foram divididos, na presente revisão, em relação aos seus desenhos metodológicos. Observou-se que a maior parte dos artigos apresentava-se com desenho teórico $(43,7 \%)$, incluindo revisões de literatura, revisões sistemáticas e revisões integrativas (Tabela 1). Estudos transversais (18,7\%) (Tabela 2) e psicométricos (25\%) (Tabela 3) também foram frequentes na amostra desta revisão. $\mathrm{O}$ delineamento com menor frequência de uso nos estudos incluídos foi o qualitativo (12,5\%) (Tabela 4). As dissertações e tese tiveram, como principal delineamento, o método transversal (54\% dos estudos), seguido do qualitativo (31\%).

Construtos variados foram investigados nos artigos encontrados nas bases de dados pesquisadas. $\mathrm{O}$ mais frequente foi o bem-estar (35,5\% dos estudos), seguido de esperança $(18,7 \%)$, discussões teóricas sobre a Psicologia Positiva de forma geral (12,5\%) e avaliação em Psicologia Positiva, excelência humana, felicidade, afetos positivos e negativos e resiliência, cada um com $6,2 \%$ dos artigos. Em relação às dissertações e tese, do total de 13 , os construtos mais frequentes foram a resiliência $(n=3)$ e o bem-estar $(n=3)$.

Em relação aos instrumentos utilizados pelos artigos incluídos nesta revisão, observou-se que escalas voltadas para a satisfação foram as mais utilizadas (incluindo diversos contextos, como satisfação de vida, no trabalho, conjugal e para estudantes). Foram incluídos, também, escalas de avaliação do bem-estar (subjetivo e disposicional), esperança, felicidade e autoestima.

Para fins de análise da produção brasileira e visão das perspectivas futuras da produção nacional na área, foram considerados também os anais do I Congresso Brasileiro de Psicologia Positiva, composto por 122

Tabela 1

Características dos Estudos Teóricos

\begin{tabular}{lcccc}
\hline \multicolumn{1}{c}{ Autores e ano } & $\begin{array}{c}\text { Tipo de } \\
\text { publicação }\end{array}$ & $\begin{array}{c}\text { Periódico (fator de impacto) } \\
\text { ou local de origem }\end{array}$ & Delineamento & $\begin{array}{c}\text { Construto } \\
\text { avaliado }\end{array}$ \\
\hline Neri (2002) & artigo & $\begin{array}{c}\text { Revista Latinoamericana de } \\
\text { Psicologia (nc) }\end{array}$ & revisão sistemática & Bem-estar subjetivo \\
$\begin{array}{l}\text { Passareli e Silva } \\
(2007)\end{array}$ & artigo & $\begin{array}{c}\text { Estudos de Psicologia - Cam- } \\
\text { pinas (nc) }\end{array}$ & revisão de literatura & Bemestar \\
$\begin{array}{l}\text { Paludo e Koller, } \\
\text { (2007) }\end{array}$ & artigo & Paidéia (nc) & revisão de literatura & Psicologia Positiva \\
$\begin{array}{l}\text { Scorsolini-Comin } \\
\text { eSantos (2010) }\end{array}$ & artigo & Psicologia Reflexão e Crítica & revisão integrativa & Avaliação em \\
$\begin{array}{l}\text { Garcia-Santos et al. } \\
\text { (2012) }\end{array}$ & artigo & Paidéia (nc) & Psicologia Positiva \\
Scorsolini-Comin, & artigo & Psicologia: Reflexão e Crítica \\
Fabio et al. (2013) & (nc) & revisão de literatura & Bem-estar \\
Santos et al. (2013) & artigo & Clinical Practice \& Epidemiol- & revisão & Estratégias de \\
& & ogy in Mental Health & Psicologia Positiva \\
Oliveira (2012) & dissertação & UNB & revisão sistemática & Bem-estar \\
\hline
\end{tabular}

Legenda: nc - não consta 
Tabela 2

Características dos Estudos Transversais

\begin{tabular}{|c|c|c|c|c|}
\hline Autores e ano & $\begin{array}{l}\text { Tipo de } \\
\text { publicação }\end{array}$ & $\begin{array}{c}\text { Periódico (fator de impacto) } \\
\text { ou local de origem }\end{array}$ & Delineamento & Construto avaliado \\
\hline $\begin{array}{l}\text { Rodrigues \& Silva } \\
\text { (2010) }\end{array}$ & artigo & Psico- USF (nc) & transversal & Felicidade \\
\hline $\begin{array}{l}\text { Scorsolini-Comin \& } \\
\text { Santos (2011) }\end{array}$ & artigo & $\begin{array}{l}\text { Psicologia: Reflexão e Crítica } \\
\text { (nc) }\end{array}$ & transversal & Bem-estar \\
\hline Alves et al. (2012) & artigo & $\begin{array}{l}\text { Rev. Latino-Am. Enfermagem } \\
\text { (nc) }\end{array}$ & transversal & Bem-estar \\
\hline Videira (2011) & dissertação & UMESP & transversal & Resiliência \\
\hline Alves (2011) & dissertação & UFU & transversal & $\begin{array}{l}\text { Bem-estar no } \\
\text { trabalho }\end{array}$ \\
\hline Onca (2011) & dissertação & UMESP & transversal & Resiliência \\
\hline Bonino (2012) & dissertação & UMESP & transversal & $\begin{array}{l}\text { Capital psicologia, } \\
\text { suporte social } \\
\text { e adesão ao } \\
\text { tratamento }\end{array}$ \\
\hline Pieroni (2012) & dissertação & UMESP & transversal & Resiliência \\
\hline Pinheiro (2011) & dissertação & PUCRS & transversal & Bem-estar \\
\hline Gottardo (2012) & dissertação & UNIVERSO & transversal & Bem-estar \\
\hline
\end{tabular}

Legenda: nc - não consta

Tabela 3

Características dos Estudos Psicométricos

\begin{tabular}{|c|c|c|c|c|}
\hline Autores e ano & $\begin{array}{c}\text { Tipo de } \\
\text { publicação }\end{array}$ & $\begin{array}{c}\text { Periódico (fator de impacto) } \\
\text { ou local de origem }\end{array}$ & Delineamento & Construto avaliado \\
\hline Pacico et al. (2011) & artigo & $\begin{array}{l}\text { Psicologia: Reflexão e Crítica } \\
\text { (nc) }\end{array}$ & $\begin{array}{l}\text { adaptação e } \\
\text { validação }\end{array}$ & Esperança \\
\hline Casas et al. (2011) & artigo & Child Ind Res (nc) & $\begin{array}{l}\text { teste de uso } \\
\text { de escalas em } \\
\text { conjunto e em } \\
\text { diferentes países }\end{array}$ & Bem-estar \\
\hline Pacico et al. (2013) & artigo & $\begin{array}{c}\text { International Journal of } \\
\text { Testing (nc) }\end{array}$ & $\begin{array}{l}\text { adaptação e } \\
\text { validação }\end{array}$ & Esperança \\
\hline Pacico et al. (2013) & artigo & $\begin{array}{l}\text { Psicologia: Reflexão e Crítica } \\
\text { (nc) }\end{array}$ & $\begin{array}{l}\text { adaptação e } \\
\text { validação }\end{array}$ & Esperança \\
\hline Bastianello (2011) & dissertação & UFRGS & $\begin{array}{l}\text { adaptação e } \\
\text { validação }\end{array}$ & Otimismo \\
\hline
\end{tabular}

Legenda: nc - não consta 
Tabela 4

Características dos Estudos Qualitativos

\begin{tabular}{lcccc}
\hline Autores e ano & $\begin{array}{c}\text { Tipo de } \\
\text { publicação }\end{array}$ & $\begin{array}{c}\text { Periódico (fator de impacto) } \\
\text { ou local de origem }\end{array}$ & Delineamento & Construto avaliado \\
\hline Lemos et al. (2009) & artigo & Ciência e Saúde Coletiva (nc) & $\begin{array}{c}\text { quantitativo } \\
\text { e qualitativo } \\
\text { fenomenológico } \\
\text { exploratório }\end{array}$ & $\begin{array}{c}\text { Afetos positivos e } \\
\text { negativos }\end{array}$ \\
Noronha et al. (2012) & artigo & Psico- USF (nc) & Análise de conteúdo \\
embasada na \\
Rocha (2011) & dissertação & UFMG & $\begin{array}{c}\text { qualitativo e } \\
\text { quantitativo }\end{array}$ & $\begin{array}{c}\text { Psicologia Positiva } \\
\text { Projetos de vida, } \\
\text { escola }\end{array}$ \\
Klein (2011) & tese & FEUSP & $\begin{array}{c}\text { qualitativo e } \\
\text { quantitativo }\end{array}$ & Motivação \\
Gomes (2012) & dissertação & PUCRS & qualitativo & qualitativo \\
Seibel (2012) & dissertação & UFRGS & Intervenção \\
& & & embasada na \\
& & & Psicologia Positiva \\
\hline
\end{tabular}

Legenda: nc - não consta

resumos voltados, especificamente, para o tema de forma geral ou de construtos específicos. Observou-se que a maior parte dos trabalhos apresentados estava relacionado com o bem-estar (14\%), o otimismo (7\%) e a criatividade $(7 \%)$, em diversos contextos (desde organizacional até enfrentamento de doenças e eventos estressores). Pôde-se, ainda, observar também que construtos, como resiliência e esperança, foram estudados de maneira conjunta em diversos estudos teóricos e empíricos, havendo uma ampliação do escopo de investigação nos estudos mais atuais, o que é possivelmente relacionado à maior disponibilidade de instrumentos de avaliação de construtos da Psicologia Positiva.

\section{Discussão}

Como objetivo principal, a presente revisão considerou os estudos nacionais, publicados até então, de modo a possibilitar o traçado de um perfil dos estudos em Psicologia Positiva no Brasil, indexados nas bases de dados selecionadas, atualizando os dados das revisões elaboradas até o momento. A primeira revisão sobre a Psicologia Positiva desenvolvida no Brasil foi desenvolvida por Yunes (2003) sobre o fenômeno da resiliência vinculado ao movimento da Psicologia Positiva. Ainda, em 2003, Junqueira e Deslandes (2003) também discutiram o conceito de resiliência a partir de uma revisão crítica. Posteriormente, Paludo e Koller (2007) em um artigo intitulado "Psicologia Positiva: uma nova abordagem para antigas questões" relataram que o primeiro manuscrito no Brasil, que avaliou um construto estudado pela Psicologia Positiva, foi de Hutz, Koller e Bandeira (1996), sobre a resiliência e vulnerabilidade em crianças em situação de risco. No entanto, na ocasião, os autores não a relacionavam diretamente com a Psicologia Positiva. Além disso, as revisões citadas anteriormente mostram em seus resultados a escassez de estudos nacionais voltados ao tema, visto que, por meio de uma busca com o termo "Psicologia Positiva", no Index Psi-Periódicos (www.bvs-psi.org.br), encontraram apenas uma referência.

Além da revisão de Paludo e Koller (2007), outras três revisões foram publicadas no ano de 2007, representando a busca por mais informações dessa nova área. Objetivando estudar construtos específicos, Salvetti e Pimenta (2007) apresentaram uma revisão crítica da literatura sobre a crença de autoeficácia relacionada à dor crônica e sobre os métodos para sua avaliação. 
Passareli e Silva (2007) e Costa e Pereira (2007) descreveram uma das principais variáveis avaliadas em Psicologia Positiva - o bem-estar subjetivo -, também reconhecido como felicidade, segundo os autores na data da publicação (Passareli \& Silva, 2007). Costa e Pereira (2007) depreenderam que o bem-estar subjetivo/felicidade pode ser percebido enquanto um traço, um estado ou "estar no controle de sua vida". Também, na presente revisão, observou-se que o bem-estar é um dos construtos mais estudados na área da Psicologia Positiva, já que a maioria dos trabalhos apresentou esse foco de estudo $(37,5 \%)$.

No entanto, esse conceito já se apresenta de forma mais ampla atualmente e, no último ano, Scorsolini-Comin, Fontaine, Koller e Santos (2013) relataram as mudanças paradigmáticas da teoria da Felicidade Autêntica (2002) para a teoria do Bem-Estar (2011), ambas desenvolvidas na Psicologia Positiva por Martin Seligman. A teoria do Bem-Estar acrescenta a realização e os relacionamentos interpessoais aos elementos já incluídos na primeira teoria (emoções positivas, engajamento, sentido), destacando que o bem-estar não dependeria apenas de aspectos individuais, mas de questões ligadas ao contexto e relacionamentos interpessoais. Enquanto a felicidade autêntica buscava a satisfação com a vida, o bem-estar almeja o florescimento, construto mais complexo e dinâmico. A teoria do Bem-Estar abre possibilidade de que se desenvolvam políticas públicas relacionadas à promoção da qualidade de vida, sem excluir a necessidade de constante revisão dessa abordagem (Scorsoline-Comin et al., 2013).

Também no último ano, uma revisão sistemática elaborada por pesquisadores de diferentes países, incluindo duas pesquisadoras brasileiras, foi além das definições e reflexões sobre a Psicologia Positiva, avaliando o papel das emoções positivas e da Psicologia Positiva no tratamento da depressão. Nos 28 artigos incluídos na referida revisão, foram observadas melhoras significativas nos sinais e sintomas de depressão, após o uso de diferentes estratégias voltadas para a Psicologia Positiva, como o aumento de emoções positivas e desenvolvimento de forças pessoais (busca de direção, significado e compromisso para a vida) (Santos et al., 2013).

Porém, antes de avaliar intervenções, percebe-se a necessidade de adaptação e validação de instrumentos para o contexto brasileiro e, no ano de 2010, Scorsoline-Comin e Santos apresentaram uma revisão integrativa da literatura voltada, exclusivamente, para os instrumentos de avaliação existentes no Brasil. Pelas bases LILACS e SciELO (1970-2008), encontraram apenas um instrumento desenvolvido em âmbito nacional, uma escala de bem-estar subjetivo inspirada em instrumentos internacionais para uma amostra específica (policiais militares do Distrito Federal) (Albuquerque \& Tróccoli, 2004). Na presente revisão, foram encontrados quatro artigos (Pacico, Zanon, Bastianello, \& Hutz, 2011; Casa et al., 2011; Pacico, Bastianello, Zanon, \& Hutz, 2013a; Pacico, Bastianello, Zanon, Repport, \& Hutz, 2013b) e uma dissertação (Bastianello, 2011) (Tabela 3) que realizaram adaptação e/ou validação de instrumentos para o contexto brasileiro. Esses estudos estiveram voltados, principalmente, para os construtos esperança, bem-estar e otimismo, demonstrando que há um crescimento considerável, desde a crítica de Scorsoline-Comin \& Santos (2010). Além disso, percebe-se que houve publicações inicialmente teóricas sobre os construtos, posteriormente de validação e adaptação de instrumento e, mais recentemente, de intervenções baseadas nos instrumentos.

Por fim, observa-se, na presente revisão, que os locais de maior concentração de realização de dissertações e teses voltadas para os temas da Psicologia Positiva são o Rio Grande do Sul e São Paulo, seguidos de Minas Gerais e Distrito Federal. Com relação a grupos de pesquisa no Brasil, destaca-se o Laboratório de Mensuração da Universidade Federal do Rio Grande do Sul (UFRGS), coordenado pelo Professor Doutor Claudio Hutz, principal centro de pesquisa em Psicologia Positiva no País, produzindo sistematicamente instrumentos de avaliação e estudos teóricos e de aplicação (Hutz, 2014). Claudio Hutz preside atualmente a Associação Brasileira de Psicologia Positiva e presidiu o I Congresso Brasileiro de Psicologia Positiva, ocorrido nos dias 8 a 10 de outubro na cidade de Porto Alegre - RS, do qual os anais foram descritos nos resultados da presente revisão. Esse evento destacou os avanços da Psicologia Positiva no Brasil, conforme a presente revisão pretende demonstrar.

Analisando, sob o enfoque da Psicologia Positiva, as explicações dos pesquisadores da excelência humana sobre o que a promove e a mantém nos mais variados domínios de atuação, conclui-se que apreciar os construtos felicidade e bem-estar nas pessoas, suas causas e seus efeitos, é importante para a construção de estratégias psicológicas. Estas devem ser centradas naquilo que faz valer a pena viver, conferindo sentido e resultando em um funcionamento humano ótimo (Garcia-Santos, 
Almeida, \& Werlang, 2012). Observou-se que esse intuito vem motivando pesquisadores brasileiros, visto o crescimento no número de publicações nos últimos anos, bem como a perspectiva de um crescimento ainda maior, o qual pode ser deduzido pelo aumento de dissertações e resumos e trabalhos apresentados no I Congresso Brasileiro de Psicologia Positiva.

\section{Conclusão}

Os artigos incluídos na presente revisão apontam para um crescente interesse, por parte dos pesquisadores, em entender essa nova área da Psicologia, constatado pelo grande número de estudos teóricos sobre o tema. É crescente, também, o esforço para adaptar e validar instrumentos para o contexto brasileiro e, mais recentemente, pela busca por avaliar intervenções na área.

Corroborando os achados de revisões anteriores, a Psicologia Positiva no Brasil está em fase de expansão, mediante o aumento dos estudos publicados na área. O estudo dos aspectos positivos do ser humano tem se tornado foco de uma série de publicações nacionais, refletindo a mudança de perspectiva dos profissionais da área da saúde sobre seus pacientes. Apesar dos grandes avanços encontrados, a literatura nacional sobre o tema ainda apresenta lacunas importantes a serem sanadas, especialmente em relação às intervenções voltadas para a Psicologia Positiva.

Observou-se, dentre os estudos incluídos e não incluídos na presente revisão, que muitos investigam construtos da Psicologia Positiva, mas não colocam esse termo como palavra-chave, o que dificulta a busca e o acesso a esses trabalhos. Cabe ressaltar ainda que o termo "Psicologia Positiva" ou "Positive Psychology", utilizado para a busca nas bases de dados, ainda não é contemplado no vocabulário estruturado DECS - Descritores em Ciências da Saúde da BIREME. Considerando que esse vocabulário foi criado para facilitar a uniformização na indexação de artigos, esse fato também pode se configurar como limitador da presente revisão. Sugere-se que mais estudos sejam desenvolvidos no Brasil, buscando esclarecer profundamente essa promissora área da Psicologia, bem como trazer para a realidade brasileira as tendências internacionais na área da Psicologia Positiva.

Por fim, constatou-se, na presente revisão, que cronologicamente foram construídos primeiro estudos teóricos, seguidos daqueles voltados para a construção de instrumentos. Atualmente, tem-se observado esforços no sentido de produzir trabalhos com foco em intervenções em Psicologia Positiva. Apesar de o Brasil ainda não estar equiparado aos grandes centros de estudos na área, como nos EUA, demonstram-se as positivas perspectivas futuras nacionais na área.

\section{Referências}

Albuquerque, A. S., \& Tróccoli, B. T. (2004). Desenvolvimento de uma escala de bem-estar subjetivo. Psicologia: Teoria e Pesquisa, 20(2), 153-164.

Alves, P. C., Neves, V. F., Coleta, M. F. D., \& Oliveira, A. F. (2012). Avaliação do bem-estar no trabalho entre profissionais de enfermagem de um hospital universitário. Revista Latino-Americana de Enfermagem, 20(4), 0104-1169.

Alves, P. C. (2011). Impacto das percepcões de suporte organizacional e social no trabalho sobre o bem-estar no trabalho da enfermagem (Dissertação de mestrado). Universidade Federal de Uberlândia - Uberlândia, MG.

Araujo, L. F. de. (2013). A psicologia positiva como fomentadora do bem-estar e da felicidade. Psicologia em Estudo, 18(4), 753-755.

Barros, R. M. A., Martín, J. I. G., \& Pinto, J. F. V. C. (2010). Investigação e prática em psicologia positiva. Psicologia Ciência e Profissão, 30(2), 318-327.

Bastianello, M. R. (2011). Adaptação e validação do teste para avaliação do otimismo LOT-R e suas relações com autoestima e personalidade (Dissertação de mestrado). Universidade Federal do Rio Grande do Sul, Porto Alegre, RS.

Bonino, M. C. P. (2012). O papel moderador do capital psicológico sobre a relação entre percepção de suporte social e adesão ao tratamento (Dissertação de mestrado). Universidade Metodista de São Paulo, São Paulo, SP.

Casas, F., Sarriera, J. C., Abs, D., Coenders, G., Alfaro, J., Saforcada, E., \& Tonon, G. (2012). Subjective indicators of personal well-being among adolescents. Performance and results for different scales in latin-language speaking countries: A contribution to the international debate. Child Indicators Research, $5,1-28$. 
Costa, L. S. M., \& Pereira, C. A. A. (2007). Bem-estar subjetivo: Aspectos conceituais. Arquivos Brasileiros de Psicologia, v. 59, n. 1, 72-80.

Garcia-Santos, S. C., Almeida, L. S., \& Werlang, B. S. G. (2012). Excelência humana: A contribuição da personalidade. Paidéia, 22(52), 251-259.

Gomes, M. A. (2012). Estudantes universitários com dificuldades de aprendizagem: Como motivá-los? (Dissertação de mestrado). Pontifícia Universidade Católica do Rio Grande do Sul, Poro Alegre, RS.

Gottardo, L. F. S. (2012). O impacto do suporte social e das avaliações autorreferentes sobre o bem-estar laboral (Dissertação de mestrado). Universidade Salgado de Oliveira, Niterói, RJ.

Hart, K. E., \& Sasso, T. (2011). Mapping the contours of contemporary positive psychology. Canadian Psychology, 52(2), 82-92.

Hutz, C. S., Koller, S. H., \& Bandeira, D. R. (1996). Resiliência e vulnerabilidade em crianças em situação de risco. Coletâneas da ANPEPP, 1(12), 79-86.

Hutz, C. S. (2014). Avaliação em psicologia positiva. Porto Alegre, RS: Artmed.

Junqueira, M. F. P. S., \& Deslandes S. F. (2003). Resiliência e maus-tratos à criança. Cadernos de Saúde Pública, 19(1), 227-235.

Klein, A. M. (2011). Projetos de vida e escola: A percepção de estudantes do ensino médio sobre a contribuição das experiências escolares aos seus projetos de vida (Tese de doutorado). Universidade de São Paulo, São Paulo, SP.

Lemos, P. M., \& Cavalcante Júnior, F. S. (2009). Psicologia de orientação positiva: Uma proposta de intervenção no trabalho com grupos em saúde mental. Ciência \& Saúde Coletiva, 14(1), 233-242.

Neri, L. A. (2002). Bienestar subjetivo en la vida adulta y en la vejez: Hacia una psicologia positiva en America Latina/Subjetive well-being in adulthood and old age: toward a positive psychology in Latin America. Revista Latinoamericana de Psicologia, 34(1/2), 55-74.

Noronha, A. P. P., \& Mansão, C. S. M. (2012). Interesses profissionais e afetos positivos e negativos: Estudo exploratório com estudantes de ensino médio. Psico-USF, 17(2), 323-331.
Oliveira, S. A. Prevenção em saúde mental no Brasil na perspectiva da literatura e de especialistas da área (Dissertação de mestrado). Universidade de Brasília. Brasília, 2012.

Onca, S. S. (2011). Resiliência em trabalhadores: Impacto da autoeficácia e da percep̧cão de suporte social (Dissertação de mestrado). Universidade Metodista de São Paulo, São Paulo, SP.

Pacico, J. C., Bastianello, M. R., Zanon, C., \& Hutz, C. S. (2013a). Adaptation and validation of the dispositional hope scale for adolescents. Psicologia: Reflexão e Crítica, 26(3), 488-492.

Pacico, J. C., Zanon, C., Bastianello, M. R., \& Hutz, C. S. (2011). Adaptation and validation of the hope index for Brazilian adolescents. Psicologia: Reflexão e Crítica, 24(4), 666-670.

Pacico, J. C., Bastianello, M. R., Zanon, C., Reppold, C. T., \& Hutz, C. S. (2013). Adaptation and validation of the Brazilian version of the hope index. International Journal of Testing, 13(3), 666-670.

Paludo, S. S., \& Koller, S. H. (2007). Psicologia positiva: Uma nova abordagem para antigas questões. Paidéia, 17(36), 9-20.

Passareli, P. M., \& Silva, J. A. (2007). Psicologia positiva e o estudo do bem-estar subjetivo. Estudos de Psicologia, 24(4), 513-517.

Pereira, C. A. A. (1997). Um panorama histórico-conceitual acerca das subdimensões de qualidade de vida e do bem-estar subjetivo. Arquivos Brasileiros de Psicologia, 49(4), 32-48.

Pieroni, J. M. (2012). Resiliência, valores bumanos e percepção de suporte social em profissionais da saúde (Dissertação de mestrado). Universidade Metodista de São Paulo, São Paulo, SP.

Pinheiro, L. B. (2011). O bem-estar na escola salesiana: Evidências da realidade (Dissertação de mestrado). Pontifícia Universidade Católica do Rio Grande do Sul, Porto Alegre, RS.

Pureza, J. R., Kuhn, C. H. C., Castro, E. K., \& Lisboa, C. S. M. (2012). Psicologia positiva no Brasil: Uma revisão sistemática da literatura. Revista Brasileira de Terapias Cognitivas, 8(2), 109-117.

Rocha, M. A. (2011). Psicologia e lazer: Um estudo sobre o tempo liberado da escola da infância contemporânea 
(Dissertação de mestrado). Universidade Federal de Minas Gerais, Belo Horizonte, MG.

Rodrigues, A., \& Silva, J. A. da (2010). O papel das características sociodemográficas na felicidade. Psico-USF, 15(1), 113-123.

Salvetti, M .G., \& Pimenta, C. A. M. (2007). Dor crônica e a crença de autoeficácia. Revista da Escola de Enfermagem da USP, 41(1), 135-40.

Santos, V., Paes, F., Pereira, V., Arias-Carrión, O., Silva, A.C., Carta, M. G., Nardi, A.E., \& Machado, S. (2013). The role of positive emotion and contributions of positive psychology in depression treatment: Systematic review. Clinical Practice \& Epidemiology in Mental Health, 9, 221-237.

Scorsolini-Comin, F., \& Santos, M. A. dos (2010). Psicologia positiva e os instrumentos de avaliação no contexto brasileiro. Psicologia: Reflexão e Crítica, 23(3), 440-448.

Scorsolini-Comin, F., Fontaine, A. M. G. V., Koller, S. H., \& Santos, M. A. (2013). From authentic happiness to well-being: The flourishing of positive psychology. Psicologia: Reflexão e Crítica, 26(4), 663-670.

Scorsolini-Comin, F., \& Santos, M. A. dos (2011). Relações entre bem-estar subjetivo e satisfação conjugal na abordagem da psicologia positiva. Psicologia: Reflexão e Crítica, 24(4), 658-665.
Seligman, M. E. P. (2002). Positive psychology, positive prevention, and positive therapy. In C. R. Snyder \& S. L. Lopez. (Eds.) Handbook of positive psychology. New York: Oxford University Press.

Seligman, M. E., \& Csikszentmihalyi, M. (2000). Positive psychology. An introduction. American Psychologist, 55(1), 5-14.

Seligman, M. E. (2004). Felicidade autêntica: Usando a nova psicologia positiva para a realização permanente. Rio de Janeiro, RJ: Objetiva.

Seligman, M. E. (2011). Florescer: Uma nova compreensão sobre a natureza da felicidade e do bem-estar. Rio de Janeiro, RJ: Objetiva.

Snyder, C. R., \& Lopez, S. J. (2009). Psicologia positiva: Uma abordagem cientifica e prática das qualidades bumanas (R. C. Costa, Trad.). São Paulo, SP: Artmed.

Videira, E. R. (2011). Impacto do conflito intragrupal, do suporte social no trabalho e do autoconceito profissional sobre a resiliência: Um estudo com policiais militares (Dissertação de mestrado). Universidade Metodista de São Paulo. São Paulo.

Yunes, M. A. M. (2003). Psicologia positiva e resiliência: $\mathrm{O}$ foco no indivíduo e na família. Psicologia em Estudo, 8, 75-84.

Recebido: 18/11/2014 $1^{a}$ reformulação: 02/01/2015

Aprovado: 12/02/2015 
Nota das autoras:

Os autores agradecem a CAPES, CNPq e FAPERGS.

Sobre as autoras:

Caroline Tozzi Reppold é professora associada I da UFCSPA, docente na graduação e pós-graduação, coordenadora do laboratório de pesquisa em Avaliação Psicológica e membro do corpo docente permanente e da comissão coordenadora do PPG Ciências da Saúde e do PPG Ciências de Reabilitação. Bolsista produtividade do CNPq.

Léia Gonçalves Gurgel é fonoaudióloga, mestre e doutoranda pelo PPG Ciências da Saúde da UFCSPA e, atualmente, é bolsista de doutorado da CAPES.

Cecilia Cesa Schiavon é nutricionista, mestre em Nutrição pela Universidade Federal de Santa Catarina, doutoranda pelo PPG Ciências da Saúde da UFCSPA e, atualmente, é bolsista de doutorado da CAPES.

\section{Contato com as autoras:}

Fundação Universidade Federal de Ciências da Saúde de Porto Alegre, Departamento de Psicologia Sarmento Leite, 245 sala 117 anexo II, Porto Alegre - RS, Brasil

CEP: 90050170

E-mail: reppold@ufcspa.edu.br. 